PROCEEDINGS OF THE

AMERICAN MATHEMATICAL SOCIETY

Volume 137, Number 3, March 2009, Pages 811-813

S 0002-9939(08)09559-2

Article electronically published on September 4, 2008

\title{
COMMENSURABILITY AND QI CLASSIFICATION OF FREE PRODUCTS OF FINITELY GENERATED ABELIAN GROUPS
}

\author{
JASON A. BEHRSTOCK, TADEUSZ JANUSZKIEWICZ, AND WALTER D. NEUMANN
}

(Communicated by Alexander N. Dranishnikov)

\begin{abstract}
We give the commensurability classifications of free products of finitely many finitely generated abelian groups. We show this coincides with the quasi-isometry classification and prove that this class of groups is quasiisometrically rigid.
\end{abstract}

The following gives the complete commensurability and quasi-isometry classification of free products of finitely generated abelian groups. The quasi-isometry classification is a special case of Papasoglu and Whyte 4 .

Theorem 1. Let $G_{i}$ be a nontrivial free product of a finite set $S_{i}$ of finitely generated abelian groups for $i=1,2$, excluding $\mathbb{Z} / 2 * \mathbb{Z} / 2$. Then the following are equivalent:

(1) The sets of ranks $\geq 2$ of groups in $S_{1}$ and $S_{2}$ are equal (the rank of a finitely generated group is the rank of its free abelian part).

(2) $G_{1}$ and $G_{2}$ are commensurable.

(3) $G_{1}$ and $G_{2}$ are quasi-isometric.

Proof. The main step is to show (1) implies (2). By going to finite index subgroups of $G_{1}$ and $G_{2}$ we can assume the groups in $S_{1}$ and $S_{2}$ are free abelian (take the kernel of the map of $G_{i}$ to a product of finite quotients of the groups in $S_{i}$ by torsionfree normal subgroups, or see the lemma below for a more general statement). Let $n_{1}=1$ and let $n_{2}, \ldots, n_{k}$ be the ranks $\geq 2$ of the groups in $S_{1}$ and in $S_{2}$. Let $r_{i}$ and $s_{i}$ be the number of rank $n_{i}$ groups in $S_{1}$ and $S_{2}$ respectively. We identify $G_{1}$ with the fundamental group of the topological space $W_{1}$ consisting of the wedge of $r_{i} n_{i}$-dimensional tori for each $i$; similarly we let $G_{2}=\pi_{1}\left(W_{2}\right)$, where $W_{2}$ is defined similarly using the $s_{i}$ 's. A finite cover of such a wedge of tori is homotopy equivalent to a wedge of tori.

We proceed in two steps. First, using finite covers we replace $\left(r_{1}, r_{2}, \ldots, r_{k}\right)$ and $\left(s_{1}, s_{2}, \ldots, s_{k}\right)$ by the sequences $\left(R_{1}, Y, Y, \ldots, Y\right)$ and $\left(S_{1}, Y, Y, \ldots, Y\right)$, respectively, where $R_{1}, S_{1}$, and $Y$ are positive integers. Then we show that, again taking finite covers, we can leave the $Y$ 's unchanged and replace both $R_{1}$ and $S_{1}$ by a positive integer $X$, making the two sequences equal and completing the argument.

Received by the editors December 6, 2007, and, in revised form, February 13, 2008.

2000 Mathematics Subject Classification. Primary 20E06, 20F65, 20F36.

This research was supported under NSF grants no. DMS-0604524, DMS-0706259, and DMS0456227 .

(C)2008 American Mathematical Society Reverts to public domain 28 years from publication 
For the first step, let $Y$ be a common multiple of $r_{2}, \ldots, r_{n}, s_{2}, \ldots, s_{n}$. We construct $W_{1}^{\prime}$ and $W_{2}^{\prime}$ in the following way. $W_{1}^{\prime}$ is a connected finite cover of $W_{1}$ obtained by appropriately gluing coverings of the constituent tori of $W_{1}$ along lifts of the base point: for each $2 \leq i \leq k$ it has $Y$ tori of dimension $n_{i}$; each $n_{i^{-}}$ dimensional torus in $W_{1}$ has $Y / r_{i} n_{i}$-dimensional tori in $W_{1}^{\prime}$ which project to it; and each torus of $W_{1}^{\prime}$ covers its image in $W_{1}$ with degree $r_{i}$. Hence $W_{1}^{\prime}$ is a covering of $W_{1}$ with degree $Y$. Similarly construct $W_{2}^{\prime}$ from $W_{2}$ using the $s_{i}$. Note that $W_{1}^{\prime}$ and $W_{2}^{\prime}$ are each homotopy equivalent to wedges of tori (by contracting an embedded tree connecting the lifts of the basepoint), but this construction doesn't control the number of 1-dimensional tori in these wedges of tori.

For step 2 we notice that, given two spaces which are homotopy equivalent to wedges of tori of dimension up to $n$, if the number of $n_{i}$-dimensional tori is the same in each for all $2 \leq i \leq k$, then the number of 1 -tori in the equivalent wedges of tori are equal if and only if the spaces have the same Euler characteristic. Now, note that a cyclic cover of degree $d$ which is a connected cover on each $n_{i}$-torus for $i \geq 2$ leaves the number of tori of dimensions $\geq 2$ unchanged. Thus, taking $W_{1}^{\prime}$ and $W_{2}^{\prime}$ as above, we can take a $\chi\left(W_{2}^{\prime}\right)$-fold cyclic cover of $W_{1}^{\prime}$ and a $\chi\left(W_{1}^{\prime}\right)$-fold cyclic cover of $W_{2}^{\prime}$ to obtain a pair of spaces homotopy equivalent to wedges of tori with the same number of tori for $2 \leq i \leq n$ and the same Euler characteristic. Hence these two covers are homotopy equivalent, thereby completing the argument.

(2) implies (3) for any finitely generated group. That (3) implies (1) follows from the easy fact that $\mathbb{Z}^{n}$ is quasi-isometric to $\mathbb{Z}^{m}$ if and only if $n=m$ plus the result of Papasoglu and Whyte [4: given two groups which are not virtually $\mathbb{Z}$ and which admit graph of groups decompositions whose edge groups are finite and whose vertex groups have one or fewer ends, they are quasi-isometric if and only if they both have the same set of quasi-isometry types of vertex groups.

The class of groups considered here is also quasi-isometrically rigid:

Theorem 2. If a group $G$ is quasi-isometric to a free product of finitely generated abelian groups, then it is commensurable with such a free product.

Proof. By the main theorem of [4] (Theorem 0.4), we have that $G$ has a graph of groups decomposition with finite edge groups and where each vertex group is quasiisometric to a finitely generated abelian group. Since a group is quasi-isometric to $\mathbb{Z}^{i}$ if and only if it has a finite-index subgroup isomorphic to $\mathbb{Z}^{i}[1,2$, 3, we claim that $G$ has a finite-index subgroup which is a free product of finitely generated free abelian groups. This follows from the lemma below.

Lemma 3. If a group $G$ splits as a finite graph of groups with finite edge groups and virtually torsion-free vertex groups $G_{v}$, then $G$ has a finite-index subgroup which is a free product of a collection of torsion-free subgroups of each of the $G_{v}$ 's together with an additional free group.

Proof. Let $K_{e}$ denote the edge group corresponding to an edge $e$. A standard classifying space $B G$ for $G$ is obtained by gluing spaces into a "graph of spaces" with spaces $B G_{v}$ at the vertices glued in the obvious way to spaces $B K_{e} \times[0,1]$ for the edges. For each $i$ let $N_{v}$ be a torsion-free normal subgoup of $G_{v}$ of index $y_{v}$ and let $Y$ be a common multiple of the $y_{v}$. If $e$ is an edge that abuts vertex $i$, then the image of $B K_{e}$ in $B G_{v}$ lifts to $y_{v} /\left|K_{e}\right|$ copies of (the contractible space) $\widetilde{B K}_{e}$ in the cover $B N_{v}$ of $B G_{v}$. Take a new graph of spaces where each $B G_{v}$ is replaced 
by $Y / y_{v}$ copies of $B N_{v}$ and each $B K_{e}$ is replaced by $Y /\left|K_{e}\right|$ copies of $\widetilde{B K}_{e} \times[0,1]$. If $e$ abuts vertex $i$, we glue $y_{v} /\left|K_{e}\right|$ copies of the edge space to each of the $Y / y_{v}$ $B N_{v}$ 's. We can clearly do this to get a space which is a connected finite cover of the original graph of spaces. It then has fundamental group a graph of groups with trivial edge groups and with vertex groups equal to copies of the $N_{v}$. This is a free product of the vertex groups and an additional free group given by the fundamental group of the underlying graph.

We note that versions of the results will hold for free products allowing other groups that have many isomorphic subgroups of finite index and that are quasiisometrically rigid up to commensurability, for example groups of the form (free) $\times \mathbb{Z}^{n}$ with $n \geq 1$, the Heisenberg group, etc.

For comparision, we remark that free products of a closely related family of groups, fundamental groups of closed hyperbolic surfaces, have very different commensurability and quasi-isometric classifications. In particular, Whyte proved that any nontrivial free products of fundamental groups of hyperbolic surfaces are quasiisometric, whereas the commensurability classification of such free products has a delicate reliance on the genus of the underlying surfaces; for details see $[5]$.

\section{REFERENCES}

1. H. Bass, The degree of polynomial growth of finitely generated nilpotent groups, Proc. London Math. Soc. 25 (1972), 603-614. MR0379672 (52:577)

2. S. M. Gersten, Quasi-isometry invariance of cohomological dimension, Comptes Rendues Acad. Sci. Paris Série 1 Math. 316 (1993), 411-416. MR1209258 (94b:20042)

3. M. Gromov, Groups of polynomial growth and expanding maps, IHES Sci. Publ. Math. 53 (1981), 53-73. MR623534 (83b:53041)

4. P. Papasoglu and K. Whyte, Quasi-isometries between groups with infinitely many ends, Comment. Math. Helv. 77 (2002), no. 1, 133-144. MR.1898396 (2003c:20049)

5. K. Whyte, Amenability, bi-Lipschitz equivalence, and the von Neumann conjecture, Duke Math. J. 99 (1999), no. 1, 93-112. MR1700742 (2001a:20064)

Department of Mathematics, Columbia University, New York, New York 10027

E-mail address: jason@math.columbia.edu

Department of Mathematics, Ohio State University, Columbus, Ohio 43210 - and Mathematical Institute of the Polish Academy of Sciences

E-mail address: tjan@math.ohio-state.edu

Department of Mathematics, Barnard College, Columbia University, New York, NEW YoRK 10027

E-mail address: neumann@math.columbia.edu 